\title{
The Research of Leaves Characteristics
}

\author{
Zhenshen Zhu ${ }^{1, a^{*}}$, Yanliu Nie ${ }^{1, b}$ and Liyun Wang ${ }^{1, c}$ \\ ${ }^{1}$ Information Engineering Department, Zhengzhou University of Industrial Technology, Xinzheng, \\ 451100, China \\ azhuzhenshen115@126.com, b361045705@qq.com, '912725921@qq.com
}

Keywords: Eigen value leaf index; BP neural networks; Multivariate analysis; Regression analysis

\begin{abstract}
The classification of leaves and the shapes of leaves related to the leaf characteristics, such as the aspect ratio is the main judgement standard of a leaf. Now, we collect the data of the leaves from trees. By building the BP neural network model, we can obtain the classification results. Then considering the veins of the leaves, gene, evolution, environmental factors, by clustering analysis, obtaining the reason why leaves have different shapes. While studying the model of the growth of the trees, it found that the branch of a tree structure on the outline of the outline of basic without the influence, so it can just research the relation between leaf form and the outline of trees,. For the collected data using linear least squares fitting, we obtained leaf form and the relationship between the tree. Through a regression model, finding that leaf form index and tree index has certain negative correlation.
\end{abstract}

\section{Introduction}

For the shape of the leaves classification, by collecting the data, to the eigen value of leaf form, the aspect ratio, the widest place such as the position of the characteristic value, through a BP neural network mathematical model, with data collected, using them as the neural network's input, and to choose appropriate output node, and training the neural network builded 500 times, applies the self- organizing feature map network to do competition learning for each sample, and then automatically to generate the different output that are relative with the classification so as to obtain the classification result. For the relation between the leaf form and the outline of a tree, through the data collected by fitting, one can get fitting curve and establish Regression Model. Then analysis of the data regression and discuss leaf form and cooked the outline of the correlation of structure and branches [1].

\section{The Classification of the Leaves}

In the plant taxonomy, the shape of the leaves, namely leaf form, the aspect ratio, the widest position, leaf base concave degree and gray and leaf crack characteristics is to judge the major indexes of the leaf form[2].

The aspect ratio, namely the leaf widths $(\mathrm{W})$ and leaf length $(\mathrm{L})$ ratio aspect ratio $=\mathrm{L} / \mathrm{W}$. The most wide position the widest place into two parts of the horizontal axis of the position of foliage, in part from the horizontal axis to determine the juncture the farthest point, as a parallel to the horizontal axis that the line (BL). Define the most wide position for $\mathrm{k} 1, \mathrm{k} 2$ to the straight distance average (L 2) and leaf length (L 1) ratio[3].

The most wide position index $=\mathrm{L} 2$ / L 1 leaf base concave degree set linear to the distance of the BL combining site for $\mathrm{L} 3$, the leaf base concave degree by next type can be said:

Base concave degree index $=\mathrm{L} 3 / \mathrm{L} 1$ gray and crack characteristics of the boundary lines, local shape (pointed to identify and leaf crack) can use zigzag leaf number, and the number of crack is quantitatively described.

Classification based on leaf form in plant system occupies an important position in the classification. The plant of traditional botany leaf form is divided into broadly ovate, ovate, lanceolate, round, oval, broadly elliptic, long oval, pour broadly ovate, obovate, oblanceolate, linear, sword, palm, heart-shaped, linear, fans, etc. 
Now we use a BP neural network mathematics model, mainly based on the traditional classification about the definition of leaf form, to the aspect ratio as the main classification feature, combined with the other features, such as the widest premises position of leaves, leaf base characteristics classification[4].

BP network's input and output is highly nonlinear mapping, the layer 3 BP neural network that can be very good to approach any continuous function. Based on the leaves of the sample, a three layers of BP network, and the BP neural network mathematics model are as follows:

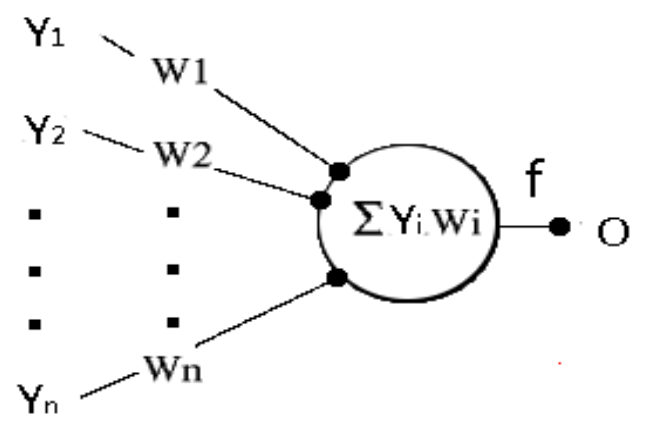

Figure 1. BP Neural Networks

(1) one will weights initialized to the random number between $0 \sim 1$;

(2) from the samples to variable W1, W2, W3, W4, input network, expect expected output E1 and $\mathrm{E} 2$

(3) among calculation hidden output $\mathrm{H} 1, \mathrm{H} 2, \mathrm{H} 3, \mathrm{Hn}$, the network and the actual output

(4) the actual output and the expectations of the error

$\delta_{k}=\left(E_{K}-O_{K}\right) \cdot O_{K} \cdot\left(1-O_{K}\right),(k=1,2)$

Hidden calculation error

$\delta_{J}^{*}=H_{J} \cdot\left(1-H_{J}\right) \cdot \sum_{k=1}^{2} \delta_{k} w_{j k},(j=1,2,3)$

(5) adjusting relevance weights

$w_{j k}^{n+1}=w_{j k}^{n}+\eta \delta_{k} H_{j}$

$v_{i j}{ }^{n+1}=v_{i j}{ }^{n}+\eta \delta_{j}^{*} I_{i}$

Among them $\eta$ In order to study the factors

(6) Using all the training sample repeated training network, many times iteration, one know weights to settle, practical trainer, define reflect the real output and expected output error square measure of value[5].

$$
E P^{*}=\frac{1}{2} \sum_{k=1}^{2}\left(E_{k}-O_{k}\right)^{2}
$$

Convergence condition

$$
\text { Error }=(1 / 15) \sum_{p=0}^{14} E P^{*}<\varepsilon
$$

$\varepsilon$ is given error range, when meet Error $<\varepsilon$, training ends.

Through computer simulation received a fairly good results. 


\section{Speaking of Profiles, Is Leaf Shape (General Characteristics) Related to Tree Profile/Branching Structure}

In the second problem, we use L system established mathematical model of the formation of the tree growth, get the outline of trees eventually form the basic similar, and the branches of the tree structure the outline of basic no effect, Therefore only considering the relation of the outline of a tree and the leaf form, the outline of the Tree[6], We adopt tree index $(\mathrm{S})=$ tree height/crown breadth, crown breadth $=$ (things crown diameter + north and south crown diameter $) / 2$. The shape of the leaves with leaf form index said (Y), leaf form index is the average of the 10 leaf.

$$
\begin{aligned}
& Y_{1}=1 / 10 \sum Y \\
& Y_{2}=\frac{\sum \mathrm{L}}{\sum \mathrm{W}}
\end{aligned}
$$

Table 1 The actual collection to ten sets of data

\begin{tabular}{|c|c|c|c|c|c|c|c|c|c|c|}
\hline ID & 1 & 2 & 3 & 4 & 5 & 6 & 7 & 8 & 9 & 10 \\
\hline S & 1.2339 & 1.2154 & 1.0511 & 1.183 & 1.2118 & 1.0653 & 1.3383 & 1.5132 & 1.0821 & 1.11764 \\
\hline Y1 & 1.8058 & 1.7914 & 1.8325 & 1.825 & 1.6688 & 1.8351 & 1.8298 & 1.6934 & 1.7883 & 1.85373 \\
\hline Y2 & 1.7996 & 1.7872 & 1.8216 & 1.824 & 1.6606 & 1.8205 & 1.8268 & 1.6796 & 1.7946 & 1.8532 \\
\hline
\end{tabular}

Establish fitting mathematical model, using linear least squares fitting Model:

Establishment of Model:

The first step: first selected a set of functions $\mathrm{r} 1(\mathrm{x}), \mathrm{r} 2(\mathrm{x}), \ldots, \mathrm{r} \mathrm{m}(\mathrm{x}), \quad \mathrm{m}<\mathrm{n}$, then

$$
f(x)=a_{1} r_{1}(x)+a_{2} r_{2}(x)+\ldots+a_{\mathrm{m}} r_{\mathrm{m}}(x)
$$

among them a1,a2, ..,am are undetermined coefficients .

The second step: make sure a1, a2, .., am standards (Method Of Least Squares) :

Make the squares of distance $\delta I$ of $n$ point $(x i, y i)$ and the curve $y=f(x)$ the least.

$$
\begin{aligned}
J\left(a_{1}, a_{2}, \cdots a_{m}\right) & =\sum_{i=1}^{n} \delta_{i}^{2}=\sum_{i=1}^{n}\left[f\left(x_{i}\right)-y_{i}\right]^{2} \\
& =\sum_{i=1}^{n}\left[\sum_{k=1}^{m} a_{k} r_{k}\left(x_{i}\right)-y_{i}\right]^{2}
\end{aligned}
$$

Among them

$$
R=\left[\begin{array}{ccc}
r_{1}\left(x_{1}\right) & \cdots & r_{m}\left(x_{1}\right) \\
\vdots & & \vdots \\
r_{1}\left(x_{n}\right) & \cdots & r_{m}\left(x_{n}\right)
\end{array}\right], a=\left[\begin{array}{c}
a_{1} \\
\vdots \\
a_{m}
\end{array}\right], y=\left[\begin{array}{c}
y_{1} \\
\vdots \\
y_{n}
\end{array}\right]
$$

Matlab for linear least squares fitting,

$Y(S)=a_{1} S^{m}+\cdots+a_{m} S+a_{m+1}$

polynomial existed program available:

$\mathrm{A}=\operatorname{polyfit}(\mathrm{x}, \mathrm{Y}, \mathrm{m})$

Take fitting effect the best result, found for 7 polynomial,

Fitting results as shown below: 


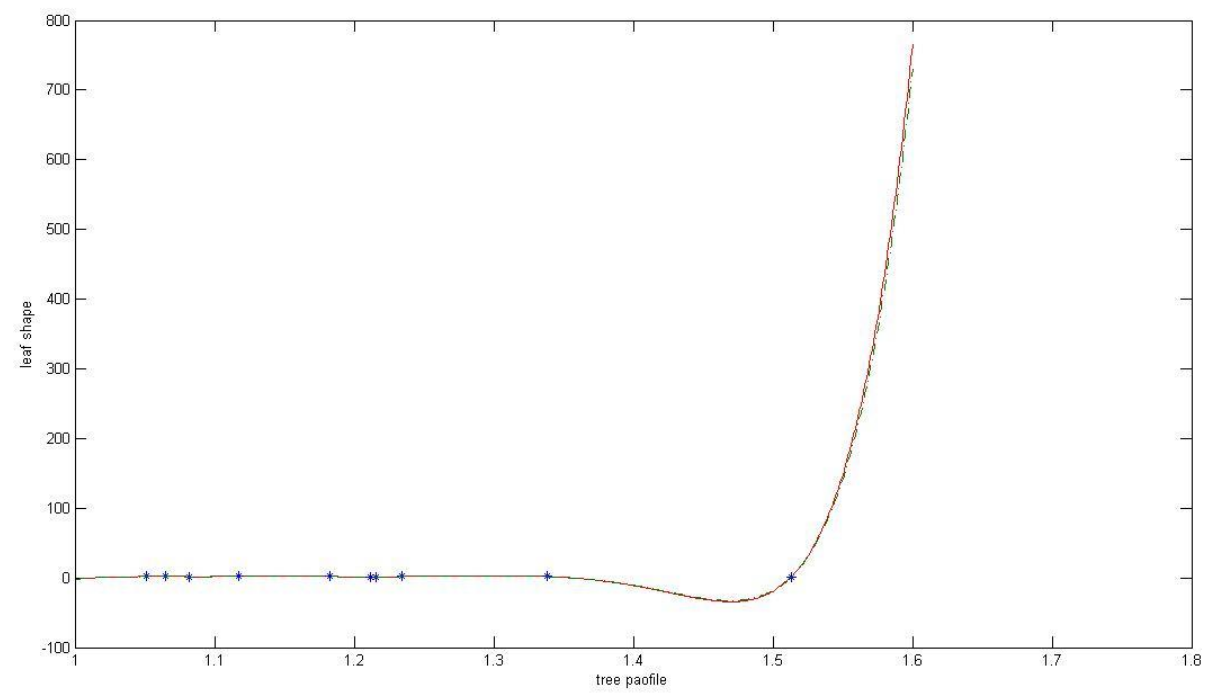

Figure 2. Fitting results

Take fitting effect the best result, found for 7 polynomial, notes for:

$Y(S)=a_{1} S^{7}+a_{2} S^{6}+a_{3} S^{5}+a_{4} S^{4}+a_{5} S^{3}+a_{6} S^{2}+a_{7} S+a_{8}$

then:

$Y(S)=0.0154 \mathrm{~S}^{7}-0.1308 \mathrm{~S}^{6}+0.4749 \mathrm{~S}^{5}-0.9557 \mathrm{~S}^{4}+1.1510 \mathrm{~S}^{3}-0.8297 \mathrm{~S}^{2}+0.3315 \mathrm{~S}-0.0566$

That leaves form and tree meet the above this relation.

A regression model,

The data obtained from the collection that leaf form index and tree index has certain negative correlation [7-10],

Linear regression model:

$Y_{1}=2.091-0.237 S$

$Y=0.7508$

Regression remaining poor

$s=\sum \sqrt{\left(\hat{Y}_{1}-Y_{1}\right)^{2} / n}=0.0296$

$Y_{2}=2.295-0.412 S$

$Y=-0.7584$

Regression remaining poor

$s=\sum \sqrt{\left(\hat{Y}_{2}-Y_{2}\right)^{2} / n}=0.03856$

From the above analysis, we can conclude that equations (1) is better than equation (2) . For

$Q Y_{1}=0.0296<Q Y_{2}=0.03856$ 
The tree leaf form of body to each index leaf form of the mean value index for good, but leaf form of the mean value index $Y_{1}$ more complicated, while it's inconvenient when leaves are too many, but $Y_{2}$ has a strong correlation with the tree [11].

\section{References}

[1] Jihyun Moon, Sarah Hake. Current Opinion in Plant Biology: How a leaf gets its shape .Volume 14, Issue 1, February 2011, Pages 24-30.

[2] Siobhan A. Braybrook andCris Kuhlemeier. How a Plant Builds Leaves. Published online before print April 2010, doi: http://dx.doi.org/10.1105/tpc.110.073924 The Plant Cell April 2010 vol. http://www.zhiwutong.com/mingci/04-15/9929.htm

[3] Tsukaya H. Annu Rev Plant Biol: Mechanism of leaf-shape determination. 2006; 57: 477-96.

[4] Wang Lanzhou, Chaizhong Lin, Ma Zhijuan, Lee Star. Geometric measurement of the leaf sequence analysis [J]. China Institute of Metrology, 2006, 17 (2): 992102. http://en.wikipedia.org/wiki/Leaf\#Large-scale_features_.28leaf_morphology.29.htm

[5] Barnsley M F. Fractals Everywhere. Boston: Academic Press Professional, 1993. 10 152. http://www.biology-online.org/11/6_leaves.htm

[6] Ogawa K, Furukawa A, Hagihara A, Abdullah AM, Awang M(1995) Morphological and phenological characteristics of leaf development of Durio zibethinus Murray (Bombacaceae).J Plant Res 108:511-515.

[7] GU Wen-zhe, Jin Wen-biao, Zhang Zhi-feng. Improved method for modeling leaf venation patterns. Computer Engineering and Applications, 2010,46 (21) : 242-245.

[8] Huang Lin, HE Peng, Wang Jing-min. JOURNAL OF NORTHWEST A\&F UNIVERSITY(NATURAL SCIENCE EDITION) .2008,36(9). 